\title{
Functional Responses in the Human Spinal Cord during Willed Motor Actions: Evidence for Side- and Rate-Dependent Activity
}

\author{
Marta Maieron, ${ }^{1,2,4 \star}$ Gian Domenico Iannetti, ${ }^{5 *}$ Jerzy Bodurka, ${ }^{2}$ Irene Tracey, ${ }^{5}$ Peter A. Bandettini, ${ }^{2,3}$ and \\ Carlo A. Porro ${ }^{1}$ \\ ${ }^{1}$ Dipartimento di Scienze Biomediche, Università di Modena e Reggio Emilia, 41100 Modena, Italy, ${ }^{2}$ Functional Magnetic Resonance Imaging Facility and \\ ${ }^{3}$ Laboratory of Brain and Cognition, National Institute of Mental Health, National Institutes of Health, Bethesda, MD 20892, ${ }^{4}$ Dipartimento di Scienze \\ Tecnologie Biomediche, Università di Udine, 33100 Udine, Italy, and 5Department of Physiology, Anatomy, and Genetics, University of Oxford, Oxford OX1 \\ 3QX, United Kingdom
}

\begin{abstract}
Although the spinal cord is the output station of the central motor system, little is known about the relationships between its functional activity and willed movement parameters in humans. We investigated here blood oxygenation level-dependent functional magnetic resonance imaging ( $\mathrm{fMRI}$ ) signal changes in the cervical spinal cord during a simple finger-to-thumb opposition task in 13 right-handed volunteers, using a dedicated array of 16 receive-only surface coils on a 3 Tesla MRI system. In a first experiment, we found significant fMRI signal increases on both sides of the lower cervical spinal cord while subjects performed the motor task at a comfortable pace $(\sim 0.5$ $\mathrm{Hz}$ ) using either hand. Both the spatial extent of movement-related clusters and peak signal increases were significantly higher on the side of the cord ipsilateral to the moving hand than on the contralateral side. Movement-related activity was consistently larger than signal fluctuations during rest. In a second experiment, we recorded spinal cord responses while the same motor sequence was performed using the dominant hand at two different rates $(\sim 0.5$ or $1 \mathrm{~Hz})$. The intensity but not the spatial extent of the response was larger during higher rates, and it was higher on the ipsilateral side of the cord. Notwithstanding the limited spatial resolving power of the adopted technique, the present results clearly indicate that the finger movement-related fMRI signals recorded from the spinal cord have a neural origin and that as a result of recent technological advances, fMRI can be used to obtain novel and quantitative physiological information on the activity of spinal circuits.
\end{abstract}

Key words: functional magnetic resonance imaging; hand; humans; cervical spinal cord; movement rate; voluntary movement

\section{Introduction}

The functional characteristics of spinal neurons involved in reflex motor responses have been extensively investigated by electrophysiological experiments in anesthetized animals (for review, see Baldissera et al., 1981; Jankowska, 1992; Fetz et al., 2000). More recently, chronic electrophysiological recordings in unanesthetized monkeys performing tracking tasks unraveled several aspects of the physiological properties of cervical spinal interneurons during the preparation and execution of hand movements (Maier et al., 1998; Perlmutter et al., 1998; Prut and Fetz, 1999; Prut and Perlmutter, 2003). As with cortical motor neurons (for review, see Evarts, 1981), activity in large populations of spinal interneurons displays a parametric relation to static torque and to rate of change of torque (Maier et al., 1998).

\footnotetext{
Received Sept. 8, 2006; revised Feb. 2, 2007; accepted Feb. 23, 2007.

This work was supported by Human Frontier Science Program Grant RGP0013/2004 (C.A.P., P.A.B., I.T.); G.D.I was supported by Pfizer United Kingdom.

${ }^{*} M . M$. and G.D.I. contributed equally to this work.

Correspondence should be addressed to Prof. Carlo A. Porro, Department of Biomedical Sciences, University of Modena and Reggio Emilia, Via Campi 287, 41100 Modena, Italy. E-mail: porro@unimore.it.

DOI:10.1523/JNEUROSCI.3910-06.2007

Copyright $\odot 2007$ Society for Neuroscience $\quad$ 0270-6474/07/274182-09\$15.00/0
}

Unlike cortical cells, they are often active during movement in opposite directions as well as at rest, and display complex relationships between their response patterns and the output effects in target muscles (Fetz et al., 2002).

So far, the activity of the human spinal cord during willed motor actions has been assessed mainly indirectly; indeed, most functional imaging studies on the human motor system have been limited to brain areas. Functional magnetic resonance imaging (fMRI) of the spinal cord is limited by severe technical challenges (Giove et al., 2004; Stroman, 2005); over the last 10 years, however, different groups have demonstrated activity changes in the cervical spinal cord during upper limb movements (Yoshizawa et al., 1996; Stroman et al., 1999, 2001; Backes et al., 2001; Madi et al., 2001; Stroman and Ryner, 2001; Ng et al., 2006) or in the lumbar spinal cord during lower limb motor tasks (Kornelsen and Stroman 2004), using fMRI.

A quantitative assessment of the spatial extent and intensity of spinal fMRI activity during unilateral motor tasks and of the relationship between fMRI signal change in spine and specific task characteristics has not been fully achieved. Yoshizawa et al. (1996) found increased fMRI signals in the intermediate and ventral zones of the lower cervical cord gray matter ipsilateral to the 
hand performing the task, during hand opening/closing in four healthy volunteers. Madi et al. (2001) investigated the fMRI responses in the spinal cord of four healthy volunteers during an isometric exercise involving the right biceps muscle (holding a series of weights from 0.026 to $1.2 \mathrm{~kg}$ in a fixed flexed-arm position). In three of four subjects, they found increased fMRI signals during the task at C5-C6 spinal levels, namely the expected rostrocaudal site for spinal motoneurons and segmental interneurons controlling the biceps muscle. In an unspecified fraction of voxels, a linear relationship (either positive or negative) was found between fMRI signals and exercise intensity.

In the present study, we aimed at investigating quantitatively the relationships between the side and repetition rate of willed motor activity and spinal fMRI signal changes. We studied the spinal activity during a simple finger-to-thumb opposition motor sequence in healthy volunteers, using a 16-channel spinal coil array designed for spinal fMRI. Preliminary results have been published in abstract form (Maieron et al., 2006).

\section{Materials and Methods}

Experimental protocol. All of the experiments were performed at the $\mathrm{Na}$ tional Institutes of Health (NIH) (Bethesda, MD). Thirteen healthy right-handed volunteers were studied (seven males and six females; age range, 21-44; mean, 32). All participants gave written informed consent, and the procedures were approved by the NIH Institutional Review Board. In a preliminary session outside the scanner room, subjects were trained to sequentially tap their fingers against the thumb in a fixed order (index, middle, ring, and little: 2-3-4-5) and to repeat the motor sequence (four taps) without interruptions at a frequency of $\sim 0.5 \mathrm{~Hz}$ (one finger tap every $0.5 \mathrm{~s}$ ) (experiment 1 ), or at both $0.5 \mathrm{~Hz}$ and the maximal frequency the subject could reach and maintain for $30 \mathrm{~s}$ (experiment 2). Task performance was assessed by evaluating the following three parameters: repetition rate, number of errors, and occurrence of mirror movements. Movement rate was defined as the number of complete cycles per second (i.e., frequency); errors were defined as the number of detectable pauses in the finger tapping and/or tapping of fingers in any sequence different from the expected (including consecutive taps of the same finger); mirror movements were defined as visible tonic contractions or phasic movements of the hand contralateral to the one the subject was instructed to move. The subjects were coached to perform the task until they were comfortable with the desired type and rate of movement.

In experiment 1 , we used a within-subject block design paradigm involving three conditions sequentially alternating every $20 \mathrm{~s}$ : rest and finger tapping of the right or left hand alternatively. Eleven volunteers were required to perform the finger opposition task at a rate of $\sim 0.5 \mathrm{~Hz}$, which was comfortable for all of them. Four 20 s periods of finger tapping were acquired for each hand; each period of movement was preceded and followed by a period of rest. This resulted in a total number of 17 periods (eight of movement and nine of rest) and a total duration of the experimental run of $340 \mathrm{~s}$. Instructions about the beginning, the end, and the side of the movement were given visually during the fMRI acquisition. An additional control fMRI run of the same length was performed in nine subjects while they were at rest. During this rest run, volunteers were asked to relax and to keep their hand still; no visual instructions were given.

Experiment 2 was performed in 12 volunteers, three of which were different from the ones involved in experiment 1 . We used a different block design paradigm involving three conditions, sequentially alternating every 30 s: rest and finger tapping of the dominant (right) hand at two rates, alternating in a fixed order. The decision of exploring different rates of movements of the dominant hand only was driven by the notion that the motor performance of the dominant hand is faster, more accurate, and shows less variability than the motor performance of the nondominant hand (Swinnen et al., 1996; Wittmann et al., 2001). The "low" frequency was $0.5 \mathrm{~Hz}$, and the "high" one was the maximal frequency the subject could reach and maintain without errors during the training session (typically, $1 \mathrm{~Hz}$ ). Six periods of finger tapping were acquired for each rate, and each period of movement was alternated with 30 s periods of rest ( 25 periods; $750 \mathrm{~s}$ in total). Instructions about the beginning, the end, and the repetition rate (low or high) of the movement were given visually during the fMRI acquisition.

Because volunteers were asked to perform the motor task without interruption within each period, the total number of taps per period was higher (approximately twice as large; see Results) in the high- than in the low-frequency condition. Therefore, we refer to rate-dependent effects, but the relative contribution of movement frequency coding versus movement quantity coding (Kim et al., 2005) cannot be determined from the present experiments.

For both experiments, volunteers were instructed to relax and remain still, to keep their arms aligned with the sides of the body, and to breath normally. During each experiment, the movement rate, the number of errors, and the occurrence of mirror movements were monitored by the same experimenter inside the scanner room.

fMRI data acquisition. The subjects were lying supine, and neck movements were reduced using head straps. Collection of spinal fMRI data were performed on a General Electric (Fairfield, CT) 3 Tesla VH/3 MRI scanner equipped with a home-built scalable digital multichannel MRI receiver (Bodurka et al., 2004).

A dedicated 16-elements receive-only surface-coils spinal array (specifically developed in collaboration with Nova Medical, Wakefield, MA) was used for detecting the MR signal, whereas a transmission body coil was used for uniform excitation. The coil array is in $4 \times 4$ receiveelements arrangement. The spinal array uses 16 ultralow impedance preamplifiers, which provide the first stage of nuclear magnetic resonance signal amplification as well as excellent individual coil isolation. All 16 signals from individual coils were further amplified, filtered, and fed into the custom-built MRI digital receiver for data acquisition and reconstruction (Bodurka et al., 2004).

Shimming was optimized manually by means of an algorithm implemented in the GE software platform, over a volume including the cervical spinal cord between the fifth cervical vertebra (C5) and the first thoracic vertebra (T1).

After shimming, structural images ( $1 \mathrm{~mm}$ thick) were acquired in the sagittal plane, parallel to the longitudinal axis of the spinal canal. The midline slice, including the center of the cord, was selected and used as a localizer to enable slice prescription of the functional images. Functional runs were acquired using blood oxygenation level-dependent (BOLD)sensitive, gradient-recalled echo planar imaging (EPI) sequence combined with sensitivity encoding (SENSE) technique (Pruessmann et al., 1999). EPI imaging parameters were as follows: repetition time (TR), $1000 \mathrm{~ms}$; echo time (TE), $32 \mathrm{~ms}$; SENSE reduction factor in phase encoding direction, 2; rectangular field of view (FOV), $18 \times 14 \mathrm{~cm}$; $144 \times 112$ image matrix, yielding an in-plane voxel size of $1.25 \times 1.25 \mathrm{~mm}$; slice thickness, $4.5 \mathrm{~mm}$; gap, $0.5 \mathrm{~mm}$. Ten oblique slices approximately perpendicular to the longitudinal axis of the spinal cord were positioned from the seventh cervical vertebra to the center of the fifth cervical vertebra (Fig. 1). The first 20 images were discarded to allow MRI signal to reach equilibrium. Individual magnitude coils images were reconstructed and combined (Pruessmann et al., 1999) with custom-made software in IDL (Research Systems, Boulder, CO). Our dedicated multichannel and sensitive MRI reception signal setup allowed us to conduct high-resolution EPI study of the spinal cord. Combination of SENSE technique with EPI allowed to have high in-plane spatial resolution with reasonable EPI readout duration ( $\sim 55 \mathrm{~ms})$. To increase available signalto-noise ratio in functional experiments, we chose to have slice thickness of $4.5 \mathrm{~mm}$. This results in the use of noncubical voxels for fMRI (Hyde et al., 2001). Because of the anatomy of the cervical enlargement of the spinal cord, which is simpler and less variable than that of other CNS structures (e.g., the cortical surface), we believe that this choice is a not major concern. Structural images were acquired with the same orientation using a fast spin-echo sequence (TR, $2000 \mathrm{~ms}$; TE, $68 \mathrm{~ms}$; FOV, 26 $\mathrm{cm} \times 26 \mathrm{~cm} ; 512 \times 256$ image matrix; slice thickness, $2 \mathrm{~mm}$ ).

fMRI data analysis. Image analysis was performed on each subject's data using FSL (www.fmrib.ox.ac.uk/fsl). An intramodal motion correction tool based on a linear image registration was used for functional 
images (Jenkinson et al., 2002). Data from one participant in experiment 2 were excluded from the analysis because of excessive motion.

The task timing was convolved with the standard gamma variate function implemented in FSL (lag, $6 \mathrm{~s}$; width, $3 \mathrm{~s}$ ), and the fMRI signal was then linearly modeled (Worsley and Friston, 1995) on a voxel-by-voxel basis using a general linear model (GLM) approach, with local autocorrelation correction (Woolrich et al., 2001). The two explanatory variables of interest were right-hand and left-hand movements for experiment 1 , low-rate and high-rate movements of the right hand for experiment 2. The estimated translation and rotation parameters were added as confounds in the model. Before statistical analysis, the mean signal value of each voxel time course was subtracted from that time course. We performed statistical analysis both on unfiltered data and following nonlinear high-pass temporal filtering (Gaussianweighted least-squares straight line fitting, with a high-pass filter cutoff of $120 \mathrm{~s}$ for experiment 1 and 180 s for experiment 2 ) and low-pass temporal filtering (low-pass filter cutoff of $2.8 \mathrm{~s}$ ), with similar results. Maps were thresholded at $p<0.001$.

For each scan of each subject, the spinal cord was segmented from surrounding tissues, in analogy with routine preprocessing steps in brain fMRI studies, by manually drawing borders of the spinal canal on the first functional image from each slice. The average number of all voxels within this spinal volume (including the 10 slices) was 977 (between-subjects range, 617-1409). The spinal region of interest (ROI) from each slice was then split into two parts, the right and the left sides of the spinal canal.

For each of the two experimental conditions (right- and left-hand finger movements in experiment 1; high and low movement rates in experiment 2), the number of voxels significantly correlated with the task in each ROI was calculated, thus providing information about the spatial extent of task-related activity. After a normalization to the $10 \mathrm{~s}$ preceding the movement onset, the mean time course from each voxel population was extracted and averaged across the corresponding movement periods (perimovement plots); this provided information about the intensity of task-related activity. The maximal intensity of activation for each condition was defined as the average of the $5 \mathrm{~s}$ time window including the highest peak (peak value $\pm 2 \mathrm{~s}$ ).

To empirically estimate the potential contribution of noise to taskrelated activity in experiment 1 , we compared the signal increases of clusters identified using the same experimental waveform (namely, a boxcar function with alternating $20 \mathrm{~s}$ "ON" and "OFF" periods) in the rest run with the signal increases of task-related clusters identified in the movement run.

Statistical analysis of fMRI signals and cinematic data. Behavioral data were analyzed by paired $t$ tests. The spatial extent and intensity of signal changes in the identified clusters were compared using repeatedmeasures ANOVA and paired $t$ test, using a commercial software package for statistical analysis (SPSS, Chicago IL). Within-subjects factors were the moving hand (right or left) and the side of the cord (ipsilateral or contralateral to the moving hand) for experiment 1 and side and rate of movement (high or low) for experiment 2. A value of $p<0.05$ was assumed as the statistical threshold.

\section{Results}

\section{Motor performances}

In experiment 1 , right-hand finger tapping was performed at an average rate of $0.6 \pm 0.4 \mathrm{~Hz}$, and the average number of errors across the four blocks was $0.7 \pm 1.4$; left-hand finger tapping was
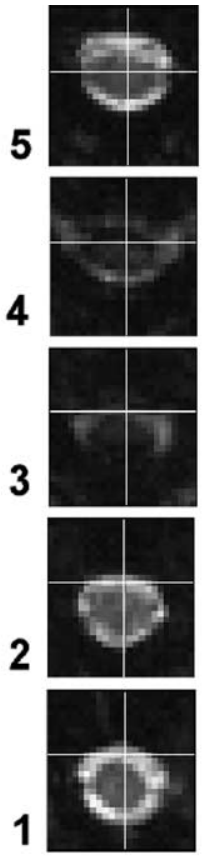

axial sections
10

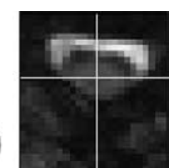

9

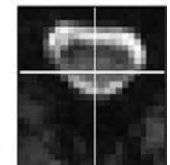

8

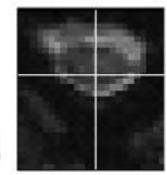

7

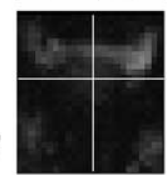

6

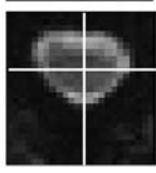

Slice prescription. Ten oblique slices perpendicular to the longitudinal axis of the spinal cord were positioned from the seventh cervical vertebra to the center of the fifth cervical vertebra (left). Slice thickness was $4.5 \mathrm{~mm}$. Note the clear contrast between the spinal cord and the surrounding cerebrospinal fluid, as well as the signal dropout at the disc/bone interface (right).

performed at an average rate of $0.5 \pm 0.3 \mathrm{~Hz}$, and the average number of errors across the four blocks was $1.5 \pm 2.3$. The difference in rate and errors between right- and left-hand movements was not significant (paired $t$ test; $p<0.1$ ). During lefthand movement only, in two subjects, mirror movements, consisting of tonic extensions of the fingers of the right hand, were detected.

In experiment 2, the motor task was executed with the right hand only. The low-rate finger tapping was performed at an average rate of $0.5 \pm 0.1 \mathrm{~Hz}$, and the average number of errors across the six blocks was $0.03 \pm 0.08$; the high-rate finger tapping was performed at an average rate of $1 \pm 0.1 \mathrm{~Hz}$, and the average number of errors across the six blocks was $0.5 \pm 0.8$. The differences in rate and errors between the two movement rates were both significant (paired $t$ test; $p<0.0001$ and $p<0.05$, respectively).

\section{Spinal cord activity related to right- or left-hand movements (experiment 1)}

In each volunteer, clusters showing signal increases related to right or left finger movements were present on both sides of the cord (both ipsilateral and contralateral to the moving hand) (Fig. 2 ). The raw time courses of fMRI signals in movement-related clusters over the whole run are shown in Figure 3, and average signal changes during the periods of right- or left-hand movement (perimovement plots) are shown in Figure 4 (top). Peak percentage increases of fMRI signals were significantly higher on the side of the cord ipsilateral to the moving hand $(3.59 \pm 0.46 \mathrm{vs}$ $2.80 \pm 0.23 \% ; t=2.62 ; p<0.05$ ) (Fig. 4, bottom). A trend toward higher increases during movements of the right hand was also observed $\left(F_{(1,10)}=4.68 ; p<0.06\right)$.

The overall spatial extent was very similar for clusters related to movements of the right or of left hand $\left[F_{(1,10)}=0.72\right.$; not significant (ns)], being on average $16.3 \%$ of all voxels within the 


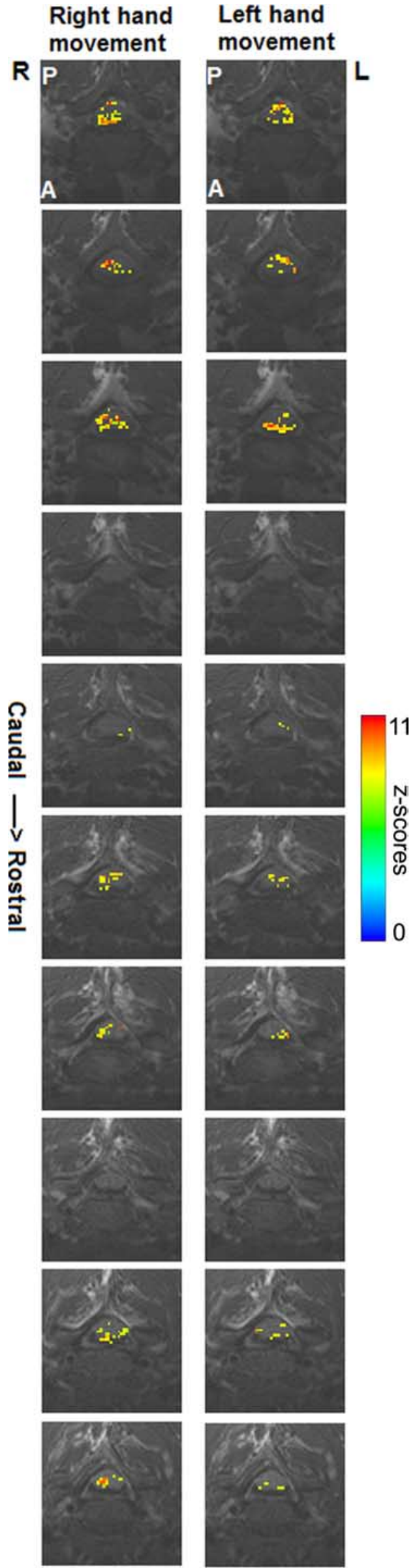

spinal ROI for right-hand movements (range in different subjects, 9-28\%), and $16.3 \%$ for left-hand movements (range, 10$26 \%)$. A significant side difference was found, with a larger extent of clusters in the hemi-spinal cord ipsilateral to the moving hand $\left(F_{(1,10)}=27.52 ; p<0.001\right)$ (Fig. 5). Approximately $40 \%$ of ipsilateral task-related clusters were also active during movements of the other hand.

Noise-related clusters identified from fMRI data of the rest run, using a boxcar waveform mimicking the experimental design (see Materials and Methods), showed much lower signal changes than those found in the same volunteers in movementrelated clusters (average signal increases over baseline during the ON periods, $0.73 \pm 0.18 \%$ vs $2.33 \pm 0.39 \% ; t=-3.98 ; p<0.01)$. The percentage spatial extent (relative to the whole spinal ROI) of noise-related clusters was also significantly lower than that of movement-related clusters $(6.3 \pm 0.71 \%$ vs $14.8 \pm 0.95 \% ; t=$ $-7.77 ; p<0.001)$.

Frequency-dependent activity during right-hand movements (experiment 2)

Maps of clusters active during movements at the two different rates are shown in Figure 6, and perimovement plots of fMRI signals in movement-related clusters are shown in Figure 7. ANOVA demonstrated a significant effect of movement rate $\left(F_{(1,10)}=6.06 ; p<0.05\right)$ and of side (with higher values on the side of the cord ipsilateral to the moving hand; $F_{(1,10)}=13.68$; $p<0.01)$ on the intensity of signal increases. On the ipsilateral side, fMRI signals related to high movement rates were significantly higher than those related to the low movement rates $\left(F_{(1,10)}=10.51 ; p<0.01\right)$. A trend toward higher signals during higher movement rates was found on the contralateral side $\left(F_{(1,10)}=3.42 ; p<0.1\right)$.

The overall spatial extent of movement-related clusters was not significantly different for the two movement rates $\left(F_{(1,10)}=\right.$ 3.09; ns) (Fig. 8). As a group average, $>60 \%$ of the identified clusters were active during finger movements at either rate (range in individual subjects, $18-89 \%$ ).

\section{Discussion}

To our knowledge, this is the first study providing quantitative evidence for a relationship between fMRI spinal cord activity and characteristics of voluntary movements in humans. Notwithstanding the limitations of the adopted approach (see below), the present results indicate that the BOLD-fMRI responses recorded from the cervical enlargement of the spinal cord have a neural origin and that fMRI can therefore be used to obtain novel physiological information on the activity of spinal circuits.

\section{Side differences in motor-related fMRI spinal signals}

We found that, during movement of either hand, the spatial extent and percentage signal increases of spinal activity were larger

$\leftarrow$

Figure 2. Experiment 1: spatial distribution of voxels displaying signal changes related to movements of the right or left hand, in the cervical spinal cord of one representative subject. Functional MRI maps are superimposed onto anatomical images. Only voxels located in the spinal ROl, identified after segmenting the spinal canal from surrounding tissues, are shown. Movement-related clusters were mostly present in the caudalmost slices, which is consistent with the known location of spinal motor circuits controlling finger movements. Maps are clearly noisy, thus preventing a detailed anatomical definition of active foci. No or a few active clusters were found in correspondence to disc/bone interface, because of the signal drop-out occurring in functional images. $R$ and $L$, Right and left sides of the body, respectively. A, Anterior, P, posterior. 
right hand movement $(\square,-)$
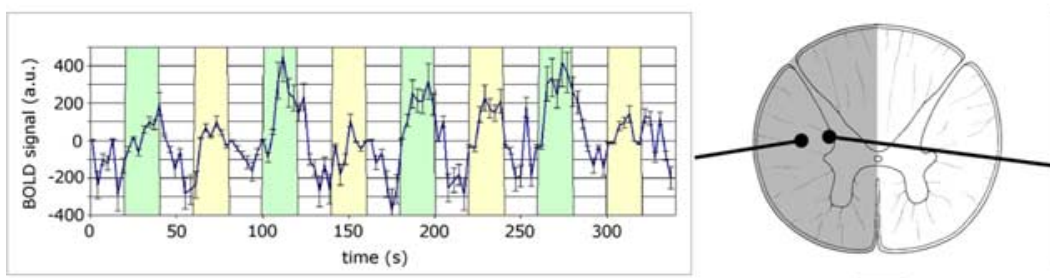

$\mathrm{R} \longleftrightarrow \mathrm{L}$

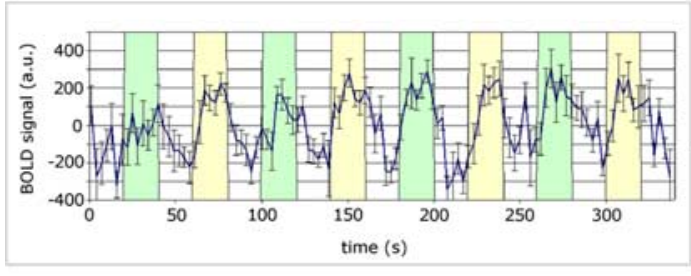

left hand movement $(\square,-)$
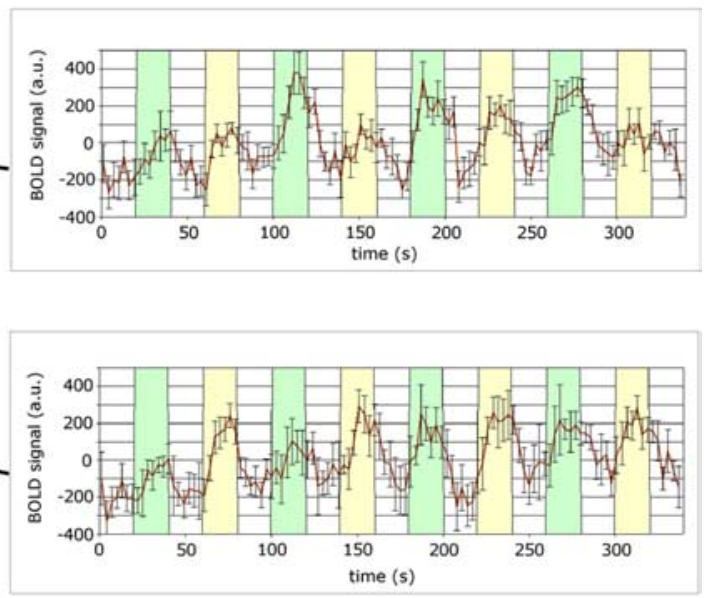

Figure 3. Experiment 1. Time profiles (mean \pm SEM of data from 11 volunteers) of unfiltered fMRI signals over the course of the experiment, in the two populations of clusters identified by the GLM analysis as related to movements of the right (blue lines) or left (red lines) hand, respectively. To improve readability, means of three consecutive time points are shown. In each side of the cord, we found clusters related to movements of either hand. a.u., Arbitrary units; L, left; $R$, right.

in the half of the spinal cord ipsilateral to the moving hand. This finding confirms and extends the results of Yoshizawa et al. (1996), who found that fMRI signals in the lower cervical cord gray matter were greater ipsilateral to the hand opening/closing than contralateral. In their study, however, changes in the contralateral (left) spinal cord were not significant.

Our observation of increased spinal fMRI signals on the side of the cord contralateral to the moving hand is intriguing. We demonstrate that contralateral signal changes are not artifactual, because they were considerably higher than noise.

To our knowledge, no study has so far described changes in the activity of primate spinal neurons on the side contralateral to the moving arm. As mentioned in the Introduction, however, the functional characteristics of spinal motor-related interneurons differ in some respects from those of cortical motor cells. For instance, a large number of spinal interneurons are active at rest and show bidirectional positive relationships between their firing rate and developed force (torque), both in the preferred and nonpreferred directions (such as wrist flexion and extension) (Maier et al., 1998; Prut and Perlmutter, 2003). Therefore, spinal interneurons seem to be ubiquitously active during a variety of movements, including those in which their postspike effects would be inappropriate (Fetz et al., 2002). This bidirectional behavior of excitatory interneurons implies that their activity is overridden by inhibitory mechanisms when their target muscles need to be inactivated. By the same token, it may be hypothesized that some interneurons discharge during movements of either (or both) hands and that their activity is inhibited by corticospinal pathways to avoid inappropriate mirror movements. It is also worth mentioning that neuronal populations responding to ipsilateral hand movements are present in the primate motor cortex (Evarts, 1966; Tanji et al., 1987, 1988) and in the human frontal and parietal cortex (see references in Porro and Corazza, 1999; Hanakawa et al., 2005).

We hypothesize that neural activity in the spinal cord contralateral to the moving hand is related to excitatory and inhibitory mechanisms involved in anticipatory postural control or in bimanual coordination (Marsden et al., 1981; Cordo and Nashner, 1982; Aruin and Latash, 1996). It has been recently shown that in sitting volunteers performing voluntary foot (Baldissera et al., 2002) or contralateral wrist (Carson et al., 2004) flexions/ extensions, the excitability of corticospinal pathways targeting the resting wrist muscles is modulated in phase with movements, whereas the H-reflex and f-waves are depressed markedly during contraction of homologous muscles of the contralateral forearm (Hortobágyi et al., 2003; Carson et al., 2004). Moreover, in standing subjects, overt electromyographic activity in forearm muscles has been described to accompany movements of the contralateral forearm (Slijper and Latash, 2000) or ipsilateral foot (Baldissera and Esposti, 2005). These complex functional changes are probably a result of highly stereotyped connections, which are therefore likely to exert their effects even under conditions in which a manifest postural intervention is not required, as when subjects lie supine in the restrained environment of the fMRI scanner.

\section{Rate-dependent changes in fMRI spinal signals}

Our results showing movement rate-dependent (or quantitydependent) increases in spinal fMRI signals extend to the spinal cord previous notions obtained by brain imaging techniques in humans. Rate-dependent increases in the BOLD fMRI signal changes indicate higher synaptic activity (in our case, either from increased descending, local circuits or peripheral afferent activity) and possibly higher discharge frequency (Logothetis et al., 2001). To our knowledge, movement rate-dependent changes in the firing patterns of spinal neurons have not been explicitly investigated in trained monkeys; however, several spinal neurons show phasic activity associated with the dynamic transition to new levels of active torque (Maier et al., 1998).

Different movement parameters are known to be encoded in the discharge of cortical motor neurons of the macaque (Schwartz, 1992; Ashe and Georgopoulos, 1994; Johnson et al., 2001), and both rate- and velocity-dependent (Turner et al., 1998) signal increases have been repeatedly described by functional imaging studies in the human brain motor networks. Ratedependent signal increases encompass several cortical areas contralateral to the moving limb that give rise to corticospinal projections (Jancke et al., 1998; Deiber et al., 1999; Peck et al., 2001; Riecker et al., 2003; Agnew et al., 2004). However, a recent event-related fMRI study suggests movement quantity coding, 

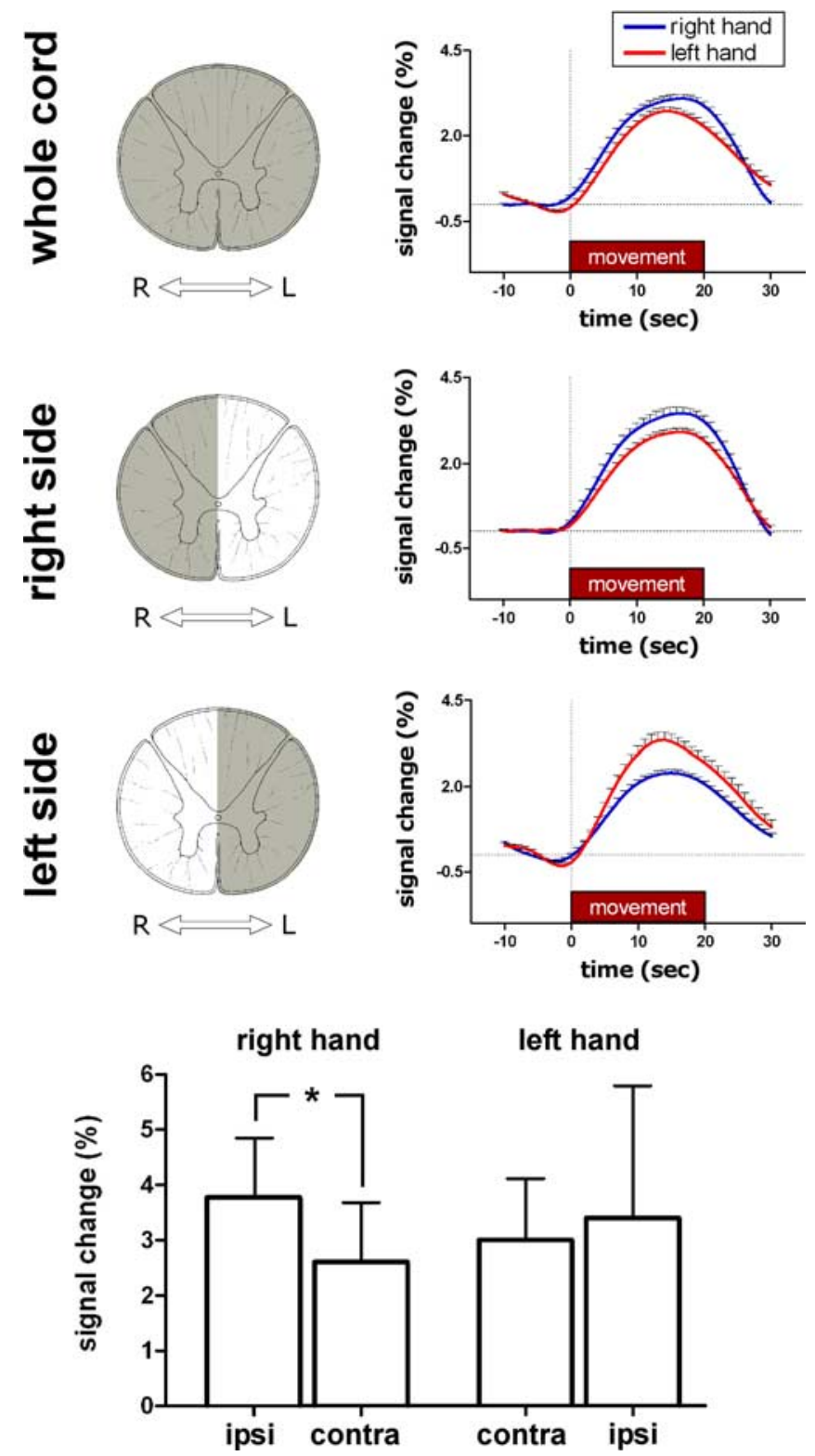

Figure 4. Movement-related fMRI signal increases (experiment 1). Top, Filtered (mean \pm SEM) time courses of fMRI signal changes from baseline, averaged around the movement (perimovement plots), in task-related clusters in the whole spinal cord, and on the right and left sides of the spinal cord. Bottom, Histograms showing percentage peak signal increases over baseline (mean \pm SEM) for clusters related to right- and left-hand movements on the two sides of the spinal cord. Asterisk indicates significant difference at $p<0.05$. contra, Contralateral; ipsi, ipsilateral; $L$, left; $R$, right.

rather than movement frequency coding, in cortical motorrelated areas (Kim et al., 2005).

In the present study, we could not detect changes in the spatial extent of the fMRI spinal response at higher movement rates. This finding does not support the hypothesis of the recruitment of additional spinal populations, and it is in contrast with the results of several mapping studies in humans, showing ratedependent increases in the spatial extent of active clusters in the contralateral sensorimotor cortex and in other motor-related areas (Schlaug et al., 1996; Jancke et al., 1998; Deiber et al., 1999; Agnew et al., 2004). It is indeed possible that our negative results are the consequence of insufficient sensitivity or spatial resolving power; however, two alternative explanations need to be men-
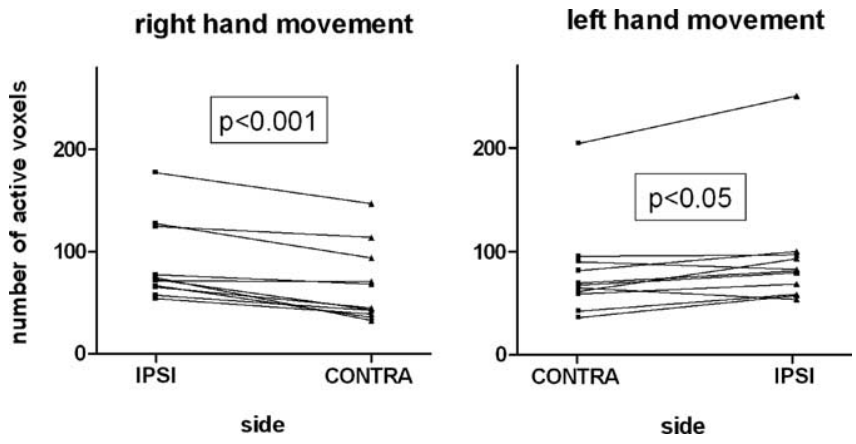

Figure 5. Spatial extent of task-related activity in experiment 1. The number of active voxels whose activity was related to movements of the right or left hand is shown, in the half of the spinal cord ipsilateral (IPSI) and contralateral (CONTRA) to the moving hand. A significant side asymmetry of task-related activity was found, with a significantly larger extent in the ipsilateral half of the spinal cord (right-hand clusters, $88 \pm 12$ vs $66 \pm 11$; paired $t=5.67 ; p<0.001$; Left-hand clusters, $93 \pm 16$ vs $79 \pm 14$; paired $t=2.75 ; p<0.05$ ).

tioned. First, in most functional imaging studies (but not in the present work), low-pass spatial filtering is applied, which may increase the size of the activated regions when the intensity of task-related signal change is increased. Second, at higher movement rates, additional neuronal aggregates can be recruited to maintain posture by keeping constant the angle of nearby and proximal joints; this implies increased activity in cortical and spinal areas, which are spatially distinct (Schlaug et al., 1996; Peck et al., 2001). Additional studies comparing spinal and brain activity in the same subjects are warranted to clarify this issue.

\section{Limitations of the study}

The technical challenges involved with performing functional MRI on the spinal cord are many (Stroman, 2005, 2006). First, the spinal cord has a small cross-section and corresponding small functional volume; its maximum diameter at the cervical level is at most 12-14 mm. Second, because of its small size and its vicinity to tissues having differing susceptibility (bone and chest cavity), magnetic field inhomogeneities predominate, creating considerable signal dropout and image distortion in standard low-resolution EPI data. High-resolution EPI as used in this study helps to reduce these effects and maintain BOLD sensitivity as long as available signal-to-noise ratio in experiments is large enough (Bellgowan et al., 2006). Third, the spinal cord is surrounded by CSF, which pulsates at the heart rate and may result in spinal cord movements, and it is immediately adjacent to the chest cavity, which expands and contracts at the rate of respiration; for these reasons, physiological noise in spinal fMRI time series is generally larger than in the cortex. Spinal cord cardiacrelated movement seems to be the most confounding effect (Stroman, 2006). Attempts to reduce this effect with cardiac gating techniques were inconclusive. Recently, postprocessing correction techniques (Glover et al., 2000) for cardiac-related and respiratory-related spinal cord movements were introduced, however, with modest improvement for cardiac-related motions (Stroman, 2006). Also, a number of papers have described the intriguing finding of a relatively strong spin-echo-related signal change in the spinal cord during activation (Stroman et al., 2001, 2002; Ng et al., 2006), suggesting that the mechanism behind functional signal changes in spinal cord could have a different origin (proton density, pure $\mathrm{T} 2$, and/or T1 changes) than the typical BOLD contrast.

In the present study, we achieved enhancement of the signalto-noise ratio by using a dedicated MRI hardware, collected 


\section{High rate}
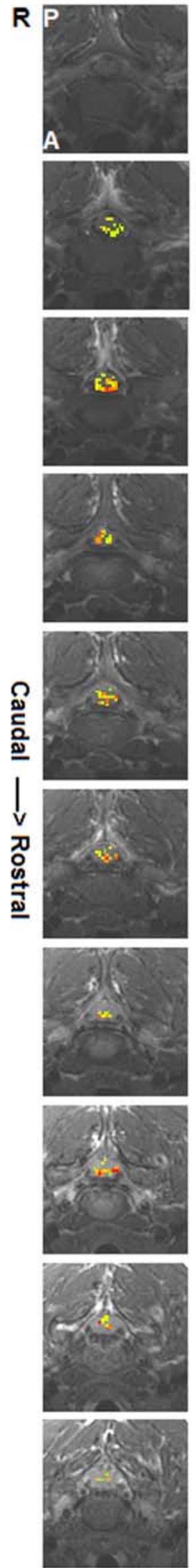

\section{Low rate}
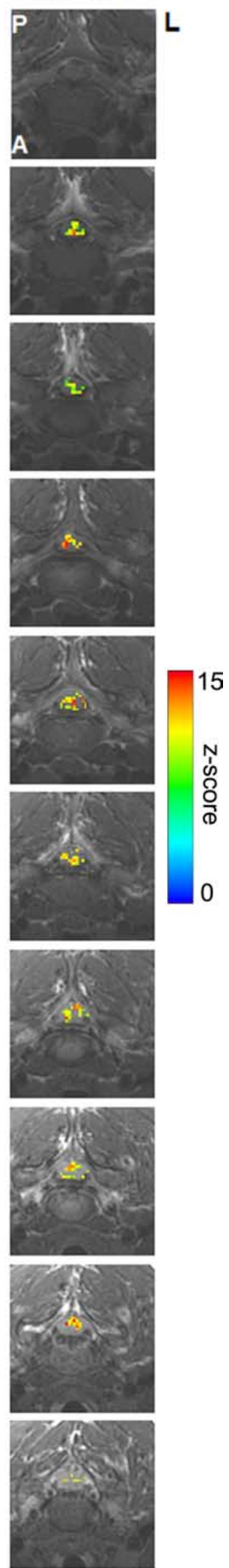

今
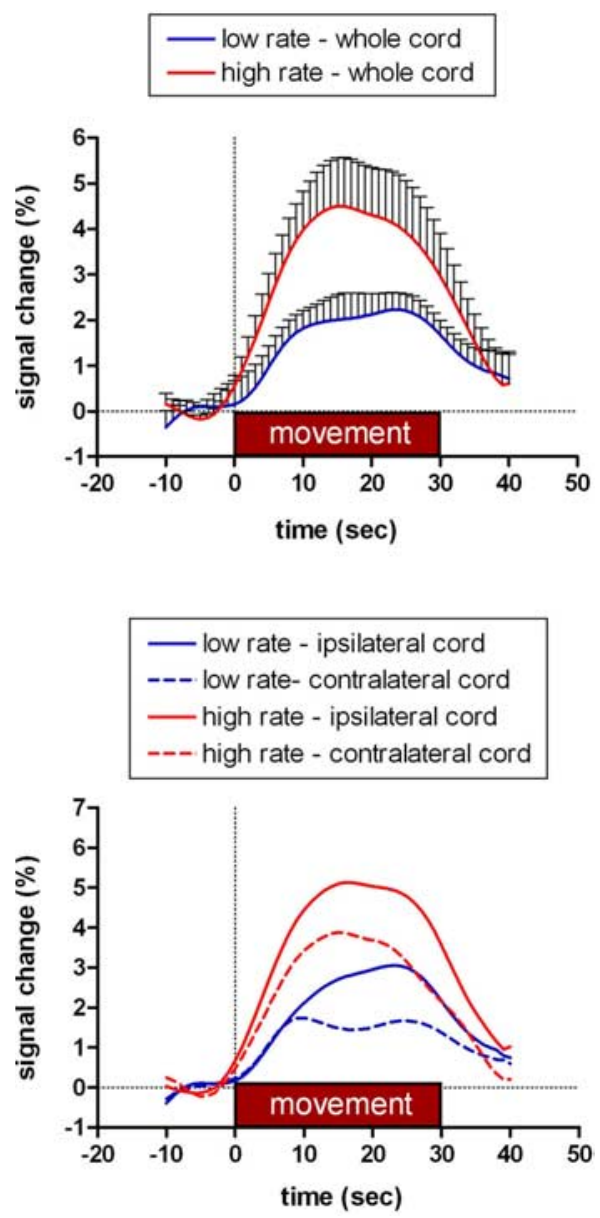

Figure 7. Perimovement plots (experiment 2). Low-pass-filtered time courses of fMRI signals averaged around the movement (perimovement plots) in clusters related to movements of the right hand at low or high frequency are shown. Note the higher signal changes during high-frequency movement. Top, Mean signals from the whole cord. Bottom, Mean signals from each hemicord.

BOLD-sensitive spinal images at high spatial resolution, and applied correction algorithms to reduce motion-related noise. However, the spatial resolving power of the adopted technique was still too coarse to investigate the fine-grain organization of the spinal cord gray matter. As a result, we quantified only leftright differences in motor-related activity. It has to be emphasized that motor-related interneurons recorded in behaving monkeys and spinal interneurons receiving cutaneous and proprioceptive input seem to be distributed widely among Rexed's laminas (Maier et al., 1998; Willis and Coggeshall, 2005). Given the simultaneous activation of motor networks and of afferent proprioceptive and tactile fibers during the finger-tapping task, it may be hypothesized that increases in spinal activity are widespread in the dorsal and ventral horns.

This hypothesis will be tested in future studies, along with additional developments in spinal cord imaging and physiological noise correction. These will prove useful to relate human

Figure 6. Spatial distribution of voxels displaying signal changes related to movements of the right hand at low or high rates, in the cervical spinal cord of one representative subject. Functional MRI maps are superimposed onto anatomical images. Only voxels located in the spinal ROl, identified after segmenting the spinal canal from surrounding tissues, are shown. Conventions are as in Figure 2. 


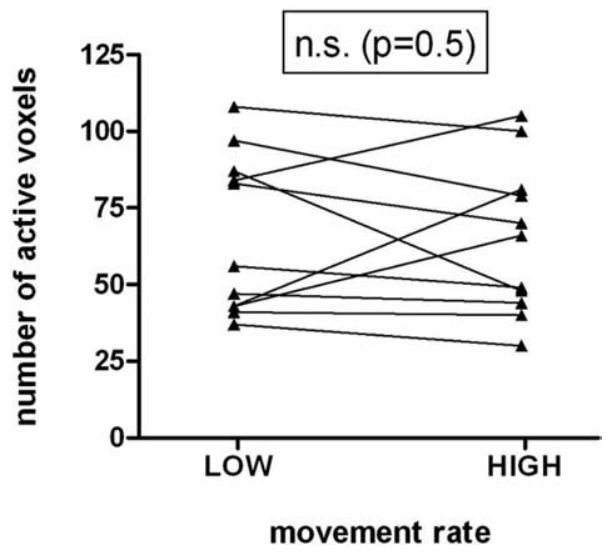

Figure 8. Spatial extent of task-related activity in experiment 2. Number of voxels in the ipsilateral side of the cord, related to low-frequency $(\sim 0.5 \mathrm{~Hz})$ and high-frequency $(\sim 1 \mathrm{~Hz})$ movements. The overall number of movement-related voxels was not significantly different for the two repetition rates.

spinal cord data to those obtained from animal models, to study spinal cord motor function under physiological and pathophysiological conditions, and to investigate the relationships between the activity of the spinal cord and brain structures involved in motor control.

\section{References}

Agnew JA, Zeffiro TA, Eden GF (2004) Left hemisphere specialization for the control of voluntary movement rate. NeuroImage 22:289-303.

Aruin AS, Latash ML (1996) Anticipatory postural adjustments during selfinitiated perturbations of different magnitude triggered by a standard motor action. Electroencephalogr Clin Neurophysiol 101:497-503.

Ashe J, Georgopoulos AP (1994) Movement parameters and neural activity in motor cortex and area 5. Cereb Cortex 4:590-600.

Backes WH, Mess WH, Wilmink JT (2001) Functional MR imaging of the cervical spinal cord by use of median nerve stimulation and fist clenching. Am J Neuroradiol 22:1854-1859.

Baldissera F, Esposti R (2005) Postural constraints to coupling of ipsilateral hand-foot movements. NeuroReport 16:1615-1619.

Baldissera F, Hultborn H, Illert M (1981) Integration in spinal neuronal systems In: Handbook of physiology. The nervous system, Vol II (Brookhart JM, Mountcastle VB, eds), pp. 509-595. Bethesda, MD: American Physiological Society.

Baldissera F, Borroni P, Cavallari P, Cerri G (2002) Excitability changes in human corticospinal projections to forearm muscles during voluntary movement of ipsilateral foot. J Physiol (Lond) 539:903-911.

Bellgowan PSF, Bandettini PA, van Gelderen P, Martin A, Bodurka J (2006) Improved BOLD detection in the medial temporal region using parallel imaging and voxel volume reduction. NeuroImage 29:1244-1251.

Bodurka J, Ledden PJ, van Gelderen P, Chu R, de Zwart JA, Morris D, Duyn JH (2004) Scalable multichannel MRI data acquisition system. Magn Reson Med 51:165-171.

Carson RG, Riek S, Mackey DC, Meichenbaum DP, Willms K, Forner M, Byblow WD (2004) Excitability changes in human forearm corticospinal projections and spinal reflex pathways during rhythmic voluntary movement of the opposite limb. J Physiol (Lond) 560:929-940.

Cordo PJ, Nashner LM (1982) Properties of postural adjustments associated with rapid arm movements. J Neurophysiol 47:287-302.

Deiber MP, Honda M, Ibanez V, Sadato N, Hallett M (1999) Mesial motor areas in self-initiated versus externally triggered movements examined with fMRI: effect of movement type and rate. J Neurophysiol 81:3065-3077.

Evarts EV (1966) Pyramidal tract neuron activity associated with a conditional hand movement in the monkey. J Neurophysiol 29:1011-1027.

Evarts EV (1981) Activity of motor cortex (MI) neurons during voluntary movement in the monkey. In: Handbook of physiology. The nervous system, Vol II (Brookhart JM, Mountcastle VB, eds.) pp. 1083-1120. Bethesda, MD: American Physiological Society.
Fetz EE, Perlmutter SI, Prut Y (2000) Functions of mammalian spinal interneurons during movement. Curr Opin Neurobiol 10:699-707.

Fetz EE, Perlmutter SI, Prut Y, Seki K, Votaw S (2002) Roles of primate spinal interneurons in preparation and execution of voluntary hand movement. Brain Res Rev 40:53-65.

Giove F, Garreffa G, Giulietti G, Mangia S, Colonnese C, Maraviglia B (2004) Issues about the fMRI of the human spinal cord. Magn Reson Imaging 22:1505-1516.

Glover GH, Li TQ, Ress D (2000) Image-based method for retrospective correction of physiological motion effects in fMRI: RETROICOR. Magn Reson Med 44:162-167.

Hanakawa T, Parikh S, Bruno MK, Hallett M (2005) Finger and face representations in the ipsilateral precentral motor areas in humans. J Neurophysiol 93:2950-2958.

Hortobágyi T, Taylor JL, Petersen NT, Russell G, Gandevia SC (2003) Changes in segmental and motor cortical output with contralateral muscle contractions and altered sensory input in humans. J. Neurophysiol 90:2451-2459.

Hyde JS, Biswal BB, Jesmanowicz A (2001) High-resolution fMRI using multislice partial k-space GR-EPI with cubic voxels. Magn Reson Med 46:114-125.

Jancke L, Peters M, Schlaug G, Posse S, Steinmetz H, Muller-Gartner H (1998) Differential magnetic resonance signal change in human sensorimotor cortex to finger movements of different rate of the dominant and subdominant hand. Cogn Brain Res 6:279-284.

Jankowska E (1992) Interneuronal relay in spinal pathways from proprioceptors. Prog Neurobiol 38:335-378.

Jenkinson M, Bannister PR, Brady JM, Smith SM (2002) Improved optimisation for the robust and accurate linear registration and motion correction of brain images. NeuroImage 17:825-841.

Johnson MTV, Mason CR, Ebner TJ (2001) Central processes for the multiparametric control of arm movements in primates. Curr Opin Neurobiol 11:684-688.

Kim JA, Eliassen JC, Sanes JN (2005) Movement quantity and frequency coding in human motor areas. J Neurophysiol 94:2504-2511.

Kornelsen J, Stroman PW (2004) fMRI of the lumbar spinal cord during a lower limb motor task. Magn Reson Med 52:411-414.

Logothetis NK, Pauls J, Augath M, Trinath T, Oeltermann A (2001) Neurophysiological investigation of the basis of the fMRI signal. Nature 412:150-157.

Madi S, Flanders AE, Vinitski S, Herbison GJ, Nissanov J (2001) Functional MR imaging of the human cervical spinal cord. Am J Neuroradiol 22:1768-1774

Maier M, Perlmutter S, Fetz EE (1998) Response patterns and force relations of monkey spinal interneurons during active wrist movement. J Neurophysiol 80:2495-2513.

Maieron M, Iannetti GD, Tracey I, Bandettini P, Porro CA (2006) A quantitative assessment of neural activity in the cervical spinal cord during finger tapping movements in humans, using fMRI. Paper presented at the 10th International Conference on Functional Mapping of the Human Brain, Florence, Italy, June 10-15. Available on CD-ROM in NeuroImage.

Marsden CD, Merton PA, Morton HB (1981) Human postural responses. Brain 104:513-534.

Ng MC, Wong KK, Li G, Lai S, Yang ES, Hu Y, Luk KD (2006) Protondensity-weighted spinal fMRI with sensorimotor stimulation at $0.2 \mathrm{~T}$. NeuroImage 29:995-999.

Peck KK, Sunderland A, Peters AM, Butterworth S, Clark P, Gowland PA (2001) Cerebral activation during a simple force production task: changes in the time course of the haemodynamic response. NeuroReport 12:2813-2816.

Perlmutter S, Maier M, FetzEE (1998) Activity and output linkages of spinal premotor interneurons during voluntary wrist movements in the monkey. J Neurophysiol 80:2475-2494.

Porro CA, Corazza R (1999) Functional magnetic resonance imaging as a tool for investigating human cortical motor function. Arch Ital Biol 137:101-114.

Pruessmann KP, Weiger M, Scheidegger MB, Boesiger P (1999) SENSE: Sensitivity encoding for fast MRI. Magn Reson Med 42:952-962.

Prut Y, Fetz EE (1999) Primate spinal interneurons show pre-movement instructed delay activity. Nature 401:590-594.

Prut Y, Perlmutter SI (2003) Firing properties of spinal interneurons during 
voluntary movement. I. State-dependent regularity of firing. J Neurosci 23:9600-9610.

Riecker A, Wildgruber D, Mathiak K, Grodd W, Ackermann H (2003) Parametric analysis of rate dependent hemodynamic response functions of cortical and subcortical brain structures during auditorily cued finger tapping: a fMRI study. NeuroImage 18:731-739.

Schlaug G, Sanes JN, Thangaraj V, Darby DG, Jancke L, Edelman RR, Warach S (1996) Cerebral activation covaries with movement rate. NeuroReport 7:879-883.

Schwartz AB (1992) Motor cortical activity during drawing movements: single-unit activity during sinusoid tracing. J Neurophysiol 68:528-541.

Slijper H, Latash M (2000) The effects of instability and additional hand support on anticipatory postural adjustments in leg, trunk, and arm muscles during standing. Exp Brain Res 135:81-93.

Stroman PW (2005) Magnetic resonance imaging of neuronal function in the spinal cord: spinal FMRI. Clin Med Res 3:146-156.

Stroman PW (2006) Discrimination of errors from neuronal activity in functional MRI of the human spinal cord by means of general linear model analysis. Magn Reson Med 56:452-456.

Stroman PW, Ryner LN (2001) Functional MRI of motor and sensory activation in the human spinal cord. Magn Reson Imaging 19:27-32.

Stroman PW, Nance PW, Ryner LN (1999) BOLD MRI of the human cervical spinal cord at 3 tesla. Magn Reson Med 42:571-576.

Stroman PW, Krause V, Malisza KL, Frankenstein UN, Tomanek B (2001) Characterization of contrast changes in functional MRI of the human spinal cord at 1.5 T. Magn Reson Imaging 19:833-838.
Stroman PW, Krause V, Malisza KL, Frankenstein UN, Tomanek B (2002) Extravascular proton-density changes as a non-BOLD component of contrast in fMRI of the human spinal cord. Magn Reson Med 48:122-127.

Swinnen SP, Jardin K, Meulenbroek R (1996) Between-limb asynchronies during bimanual coordination: effects of manual dominance and attentional cueing. Neuropsychologia 34:1203-1213.

Tanji J, Okano K, Sato KC (1987) Relation of neurons in the nonprimary motor cortex to bilateral hand movement. Nature 327:618-620.

Tanji J, Okano K, Sato KC (1988) Neuronal activity in cortical motor areas related to ipsilateral, contralateral, and bilateral digit movements of the monkey. J Neurophysiol 60:325-343.

Turner RS, Grafton ST, Votaw JR, Delong MR, Hoffman JM (1998) Motor subcircuits mediating the control of movement velocity: a PET study. J Neurophysiol 80:2162-2176.

Willis WD, Coggeshall RE (2005) Sensory mechanisms of the spinal cord, Ed 3, Vol 2. New York: Kluwer.

Wittmann M, von Steinbuchel N, Szelag E (2001) Hemispheric specialisation for self-paced motor sequences. Cogn Brain Res 10:341-344.

Woolrich MW, Ripley BD, Brady M, Smith SM (2001) Temporal autocorrelation in univariate linear modeling of fMRI data. NeuroImage 14:1370-1386.

Worsley KJ, Friston KJ (1995) Analysis of fMRI time-series revisitedagain. NeuroImage 2:173-181.

Yoshizawa T, Nose T, Moore GJ, Sillerud LO (1996) Functional magnetic resonance imaging of motor activation in the human cervical spinal cord. NeuroImage 4:174-182. 